\title{
Metallographic Investigations of Solidification Microstructures and Melt-Pool Characteristics in Oriented Single Crystals of Stainless Steel
}

\author{
L. A. Boatner*, J. B. Sipf*, S. A. David*, and Michel Rappaz**
}

*Condensed Matter Sciences and Metal and Ceramics Divisions, Oak Ridge National Laboratory, MS-6056, 1 Bethel Valley Road, Oak Ridge, Tennessee 37831

**Ecole Polytechnique Federale de Lausanne, Laboratoire de Metallurgie Physique, MX-G Ecublens, CH-1015 Lausanne, Switzerland

The joining of materials through the mechanism of "fusion welding" has long represented one of the most important industrial manufacturing methods that spans the automotive, aerospace, civil engineering, and other production and construction fields. In the fusion welding process the joining of two materials is generally accomplished by heating above their melting points a common boundary region between the two components that are to be joined. Subsequent cooling and solidification of the molten region between the two materials then forms a solid mechanical bond that joins the components. The microstructural characteristics of the region that represents the joint between the two materials that have been welded are a result of the combined heating, melting, and resolidification processes. These microstructural characteristics, in turn, govern the mechanical properties and strength of the weld. A variety of factors associated with the welding process such as the type of heating used (e.g. gas tungsten arc, electron beam, laser, etc), the welding atmosphere, the power input, and the traversing speed of the heat source determine the microstructural properties of the weld region. The purity of the components, their phase properties, and their initial microstructural characteristics are also factors that affect the final microstructure of the "fusion" and nearby so-called "heat-affected" zones of a weld. In particular, the size and relative crystallographic orientations of microstructural grains in the component materials being joined play an important role in the grain-growth and grain-selection processes that take place during the solidification of the melt pool. During the solidification phase of welding, epitaxial growth of the solidification "cells" is initiated at the interface between the sold and liquid phases that defines the weld pool shape. Accordingly, the properties of the solidification microstructure are determined primarily by the relative orientations of the melt-pool interface and the crystallographic orientation of the solid portions of the grains on which epitaxial growth occurs and the heat flow direction. The growth of the solidification "cells" or dendrites generally proceeds along so-called "easy growth" directions as determined by the system's crystallographic properties. In the work reported here, a fully austenitic face-centered-cubic ternary "stainless steel" alloy with a composition of $70 \% \mathrm{Fe}-15 \% \mathrm{Ni}-15 \% \mathrm{Cr}$ (by weight) was employed in most of the experiments. For this material, the $<100>$ crystallographic axes represent the "easy-growth" directions of the solidification cells.

In order to clearly delineate the important role played by crystallography in determining weld microstructures, we have employed oriented single crystals of the ternary Fe-NI-Cr alloy in a series of welding experiments using different heat sources - including electron-beam heating and gas tungsten arcs [1-2]. Prior to these single-crystal investigations of weld microstructures, most studies were carried out using polycrystalline metals and alloys - frequently commercial materials that were characterized by a variety of impurity levels and types and by a multiplicity of grain orientations and grain sizes. When melt pools formed using such complex systems solidify, the epitaxial nucleation and growth of the solidification cells occurs on randomly oriented grains of varying sizes and the resulting microstructures are so complex that they are not really amenable to quantification. By using single crystal specimens we have been able to develop a metallographic approach that results in 
quantifiable and reproducible microstructures in which the roles played by crystallographic effects and heat flow can be clearly delineated. The present work extends our prior studies that employed moving heat sources and focuses on the case of the re-solidification of melt pools formed in single crystals of stainless steel by a stationary heat source using the configuration illustrated in Figure 1. This single-crystal approach yields microstructures of the type illustrated in the metallographs shown in Figs 2 and 3. By employing a series of such micrographs obtained at various regions of the overall microstructure it is possible to carry out a full three-dimensional reconstruction of the solidification microstructure for stationary melt pools formed using the single-crystal ternary alloy. A reconstruction of this type is shown in figure 4 for the case of a melt pool formed on a [100]-oriented single crystal. Other features of melt pools created using single crystals such as the melt-pool surface morphology will be discussed.

\section{References}

[1] M. Rappaz, S. A. David, J. M. Vitek, and L. A. Boatner, Metall. Trans. A (Phys. Metall. Mater. Sci.) 21A, (1990) 1767.

[2] S. A. David, J. M. Vitek, M. Rappaz, and L. A. Boatner, Metall. Trans. A (Phys. Metall. Mater. Sci.) 21A, (1990) 1753.

[3] Research sponsored by the Division of Materials Sciences and Engineering, Office of Basic Energy Sciences, U.S. Department of Energy, under contract DE-AC05OR22725 with Oak Ridge National Laboratory, managed and operated by UT-Battelle, LLC.

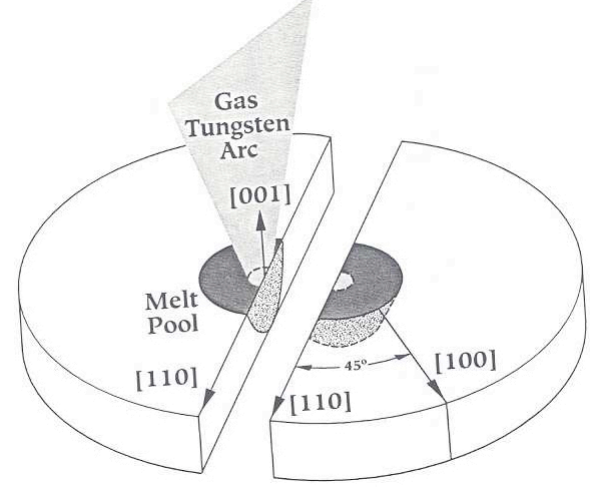

Figure 1. Stationary GTA melt pool formation on a stainless steel single crystal

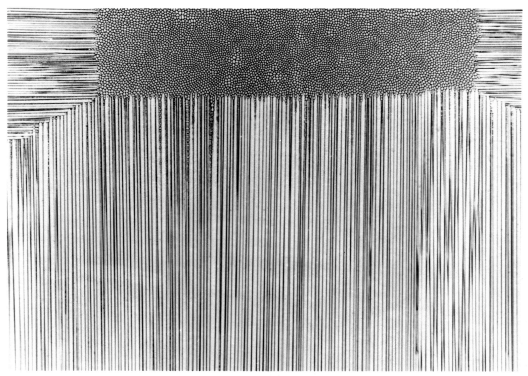

Figure 3. Top view, center section microstructure in a (100) melt pool

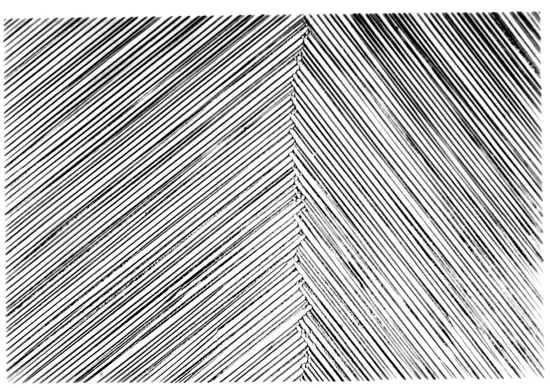

Figure 2. Transverse microstructure in a (110) single crystal melt pool

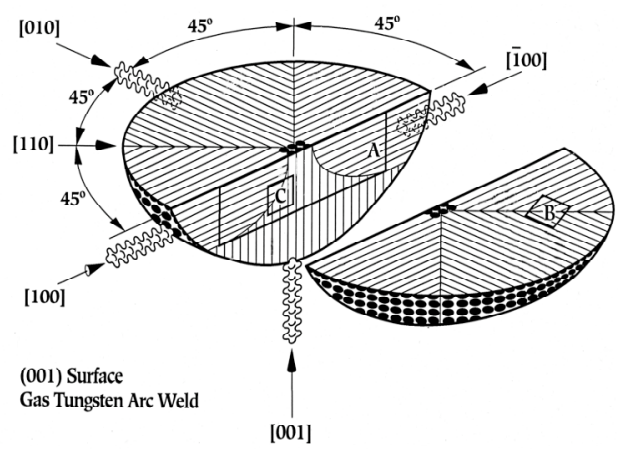

Figure 4. Three-dimensional reconstruction of solidification microstructure for a (100) single crystal melt pool 